\section{Retinopathy in the Era of Routine Hydroxychloroquine Monitoring}

\section{To the Editor:}

Hydroxychloroquine (HCQ) is a vital component of systemic lupus erythematosus (SLE) treatment, but it carries a significant risk of irreversible blindness from HCQ retinopathy. HCQ retinopathy is relatively rare, but new, more sensitive diagnostic techniques report a prevalence of up to $7.5 \%{ }^{1}$. Moreover, HCQ retinopathy may progress even after cessation of therapy ${ }^{2}$, so early detection and primary prevention are critical. Currently, primary prevention consists of controlling the daily dose and/or cumulative dose to prevent overdosage ${ }^{3}$. We therefore read with great interest "Hydroxychloroquine Blood Levels in Systemic Lupus Erythematosus: Clarifying Dosing Controversies and Improving Adherence" by Durcan, et $a l^{4}$ in The Journal of Rheumatology. We point out the implications of their findings for the use of weight-based dosing and the evidence base for HCQ retinopathy prevalence.

In their study, Durcan, et al described how regularly measuring blood levels of HCQ in patients with SLE resulted in a higher proportion of patients having blood levels of HCQ within a therapeutic range of 500-2000 $\mathrm{ng} / \mathrm{ml}$ of blood, and concluded that measuring routine blood levels of HCQ, coupled with counseling, is an effective means to ensure compliance with HCQ to improve longterm outcomes ${ }^{4}$. In addition, Durcan, et al endorsed an actual weight-based calculation to determine the starting dose of HCQ based on the observation that compliance alone, and not dose adjustment, was sufficient to bring the majority of patients into a therapeutic range $\mathrm{e}^{4}$.

Body weight-based dosing is an ongoing controversy in the ophthalmology literature. The current guidelines recommend using ideal body weight for certain individuals because it avoids the most risk ${ }^{3}$. Based on the reasoning that HCQ is not stored in fatty tissues so dosing based on actual body weight may result in overdosage for short or obese individuals, the latest guidelines recommend that short individuals be dosed at no more than $6.5 \mathrm{mg} / \mathrm{kg}$ of ideal body weight per day and that obese individuals be dosed based on height ${ }^{3}$. In fact, 2 retrospective studies reported that in patients taking HCQ, if ideal body weight were used to recalculate HCQ dose, then about half of these patients would be overdosed ${ }^{5,6}$.

However, empiric data has not uniformly supported the hypothesis that ideal body weight is a superior predictor of risk of HCQ retinopathy. A retrospective study of 2361 patients taking HCQ found that actual body weight correlated with risk of retinopathy ${ }^{1}$. In a similar fashion, a prospective study of 300 patients taking HCQ found that actual body weight strongly correlated with blood levels of $\mathrm{HCQ}^{7}$, a finding that is corroborated by the study by Durcan, et $a l^{4}$. As stated in the editorial, no study has yet definitively linked high blood concentration of HCQ to retinal toxicity ${ }^{8}$, but higher doses and longer durations of therapy have been linked to risk for HCQ retinopathy ${ }^{1,9}$, suggesting that cumulative dose may be a significant risk factor for HCQ retinopathy.

The findings by Durcan, et al also have implications for prevalence and risk of HCQ retinopathy. Namely, their results suggest that previous estimates of HCQ retinopathy prevalence may be unreliable. Importantly, Durcan, et al reported that their baseline rate of noncompliance was as high as $44 \%$, which is consistent with the literature at large ${ }^{10}$. Traditional studies reporting a prevalence of about $1 \%$ did not adequately account for patient noncompliance. Therefore, these older studies may have underestimated the risk for HCQ retinopathy. This issue is addressed in the study by Melles and Marmor, which did account for patient compliance by using pharmacy refill rates (finding average use values that were about $20 \%$ lower than the prescribed dosage $)^{1}$. However, their study relied on more sensitive diagnostic ophthalmic imaging techniques to define HCQ retinopathy, thus making direct comparison between newer and older prevalence values difficult.

Despite these difficulties, it is undeniable that, if nearly half of patients prescribed HCQ are not routinely taking it, the incidence of HCQ toxicity is most likely grossly underappreciated. This is important because the findings by Durcan, et al and the accompanying editorial suggest that we may be on the cusp of a paradigm shift. If routine blood levels are a cost-effective tool to increase compliance and therefore improve outcomes for patients with SLE, it is likely that this practice will become routine. If compliance with HCQ increases across medical practices, so too will the effective daily and cumulative doses, and with it, the risk for HCQ retinopathy. Therefore, we urge continued communication between rheumatologists and ophthalmologists to maximize care for patients with SLE and avoid risks of HCQ as we enter an era of routine HCQ monitoring.

ERIC WEINLANDER, BA, Department of Ophthalmology and Visual Sciences, University of Wisconsin-Madison, Madison, Wisconsin, USA; ALEXANDER L. RINGEISEN, MD, Department of Ophthalmology and Visual Sciences, University of Wisconsin-Madison, Madison, Wisconsin, USA; MIHAI MITITELU, MD, Department of Ophthalmology and Visual Sciences, University of Wisconsin-Madison, Madison, Wisconsin, USA. Address correspondence to E. Weinlander, Department of Ophthalmology and Visual Sciences, University of Wisconsin-Madison, 2870 University Avenue, Suite 206, Madison, Wisconsin 53705, USA.

E-mail: eweinlander@wisc.edu

\section{REFERENCES}

1. Melles RB, Marmor MF. The risk of toxic retinopathy in patients on long-term hydroxychloroquine therapy. JAMA Ophthalmol 2014;132:1453-60.

2. Mititelu M, Wong BJ, Brenner M, Bryar PJ, Jampol LM, Fawzi AA Progression of hydroxychloroquine toxic effects after drug therapy cessation: new evidence from multimodal imaging. JAMA Ophthalmol 2013;131:1187-97.

3. Marmor MF, Kellner U, Lai TY, Lyons JS, Mieler WF; American Academy of Ophthalmology. Revised recommendations on screening for chloroquine and hydroxychloroquine retinopathy. Ophthalmology 2011;118:415-22.

4. Durcan L, Clarke WA, Magder LS, Petri M. Hydroxychloroquine blood levels in systemic lupus erythematosus: clarifying dosing controversies and improving adherence. J Rheumatol 2015;42:2092-7.

5. Michaelides M, Stover NB, Francis PJ, Weleber RG. Retinal toxicity associated with hydroxychloroquine and chloroquine: risk factors, screening, and progression despite cessation of therapy. Arch Ophthalmol 2011;129:30-9.

6. Walvick MD, Walvick MP, Tongson E, $\mathrm{Ngo} \mathrm{CH}$. Hydroxychloroquine: lean body weight dosing. Ophthalmology 2011;118:2100; author reply 2101.

7. Francès C, Cosnes A, Duhaut P, Zahr N, Soutou B, Ingen-Housz-Oro $\mathrm{S}$, et al. Low blood concentration of hydroxychloroquine in patients with refractory cutaneous lupus erythematosus: a French multicenter prospective study. Arch Dermatol 2012;148:479-84.

8. Costedoat-Chalumeau N, Le Guern V, Piette JC. Routine hydroxychloroquine blood concentration measurement in systemic lupus erythematosus reaches adulthood. J Rheumatol 2015;42:1997-9.

9. Wolfe F, Marmor MF. Rates and predictors of hydroxychloroquine retinal toxicity in patients with rheumatoid arthritis and systemic lupus erythematosus. Arthritis Care Res 2010;62:775-84.

10. Costedoat-Chalumeau N, Pouchot J, Guettrot-Imbert G, Le Guern V, Leroux G, Marra D, et al. Adherence to treatment in systemic lupus erythematosus patients. Best Pract Res Clin Rheumatol 2013;27:329-40

J Rheumatol 2016;43:6; doi:10.3899/jrheum.151436 